\title{
Hybrid Systems: From Verification to Falsification
}

\author{
Erion Plaku, Lydia E. Kavraki, and Moshe Y. Vardi \\ Department of Computer Science, Rice University \\ Houston TX 77005, USA \\ \{plakue, kavraki, vardi\}@cs.rice.edu
}

\begin{abstract}
We propose HyDICE, Hybrid DIscrete Continuous Exploration, a multi-layered approach for hybrid-system testing that integrates continuous sampling-based robot motion planning with discrete searching. The discrete search uses the discrete transitions of the hybrid system and coarse-grained decompositions of the continuous state spaces or related projections to guide the motion planner during the search for witness trajectories. Experiments presented in this paper, using a hybrid system inspired by robot motion planning and with nonlinear dynamics associated with each of several thousand modes, provide an initial validation of HyDICE and demonstrate its promise as a hybrid-system testing method. Comparisons to related work show computational speedups of up to two orders of magnitude.
\end{abstract}

\section{Introduction}

Hybrid systems play an increasingly important role in transportation networks [1], manufacturing processes [2, robotics [3], and medicine and biology [4,5]. Today we find hybrid systems in sophisticated embedded controllers used in the automotive and airplane industry, and also in medical devices that monitor serious health conditions. Recently, it has been shown that hybrid systems are a powerful tool for modeling biological processes and for analyzing how complex systems, such as living organisms, survive [5].

Hybrid systems are formal models that combine discrete and continuous dynamics by associating continuous dynamics with each operating mode, while using discrete logic to switch between operating modes. For example, a hybrid system may model a vehicle whose underlying motion dynamics varies discretely depending on terrain conditions. As another example, a hybrid system may model air-traffic control, where the modes correspond to the cruising of the planes and the discrete logic models conflict-resolution protocols.

As hybrid systems model more and more complex behaviors, and as they are often part of devices operating in safety-critical situations, the verification of safety properties becomes increasingly important. A hybrid system is considered safe if unsafe states cannot be reached from initial safe states. In general, hybridsystem verification consists of formally guaranteeing that a certain property is

W. Damm and H. Hermanns (Eds.): CAV 2007, LNCS 4590, pp. 46\$-476, 2007.
(C) Springer-Verlag Berlin Heidelberg 2007 
true for the system. A rich theory exists for this problem [6, 7, 8, 9, 10, as well as several tools, such as CHECKMATE1, HYTECH2 2 , and PHAVER 3 .

Unfortunately, even for safety properties, where verification is equivalent to reachability checking, decidability holds only for hybrid systems with very simple continuous dynamics (essentially some types of linear dynamics) [1]. To handle more general hybrid systems, tools have to resort to overapproximation techniques, surveyed in [12,13. Such techniques are semi-decidable, since when a hybrid system is unsafe it may not be possible to show that unsafe states are reachable. Other recent approaches are semi-decidable in the opposite direction: capable of finding unsafe behaviors when the system is unsafe, but unable to determine that a system is safe [14, 15. In essence, the focus in these recent approaches shifts from verification to falsification, which often is the main focus of model checking in industrial applications [16.

In this work we study the following problem: Can we produce a hybrid-system trajectory from a safe state to an unsafe state when such trajectories exist? This problem is commonly known as hybrid-system testing. When a hybrid system is safe, it may not be possible to prove that unsafe states are unreachable. Such an approach trades completeness for the ability to discover safety violations for complex systems that current verification methods cannot handle. Under appropriate conditions and for certain classes of algorithms, as discussed later in the paper, as the running time increases, we can also increase our confidence in the safety of the system, since the testing method has not been able to produce a trajectory that violates safety properties. An efficient framework for finding trajectories to unsafe states can shed light on the operation of the hybrid system and may suggest possible interventions. Such framework is particularly useful in the early stages of hybrid-system development, when errors in design are common.

This work approaches hybrid-system testing using a robotics-inspired method. Initially, we exploit the insight that hybrid-system testing is in many respects related to robot motion planning. The motion-planning problem consists of searching a continuous space for a trajectory for a robotic system from an initial to a final state, such that kinodynamic constraints on the robot motion are respected and collision with obstacles are avoided [17, 18. Hybrid-system testing is also a reachability analysis on the state space of the hybrid system. In particular, finding a trajectory from a safe to an unsafe state in a hybrid system entails searching a high-dimensional state space with continuous and discrete components.

The connection with motion planning becomes deeper when we consider stateof-the art motion-planning algorithms as the starting point for the methods used for searching the continuous state space of a hybrid system. Recent advances in motion planning have made it possible to efficiently find trajectories from initial to final states even for continuous systems with hundreds of dimensions whose motion is governed by nonlinear dynamics $[17,18,19,20,21,22,23,24$.

\footnotetext{
${ }^{1}$ http://www.ece.cmu.edu/ $\sim$ webk/checkmate/

${ }^{2}$ http://embedded.eecs.berkeley.edu/research/hytech/

${ }^{3}$ http://www.cs.ru.nl/ goranf/
} 
The most successful planning methods are numerical, sampling-based methods. These methods generate samples in the state space and connect them with simple trajectories. (e.g., PRM 25], RRT [19], EST [20], PDST [22, GRIP [23], DSLX-Plan 24, and others 17, 18]). Motion planning methods, such as RRT, have already been used for hybrid-system testing for nonlinear hybrid systems with few modes [15, 14 .

Departing from traditional robot motion planning, we introduce a discrete component to our work that is responsible for managing the potentially huge complexity of the discrete transitions. The contribution of this work is the development of a multi-layered framework for hybrid-system testing that blends sampling-based motion planning with discrete searching. The motivation and many of our design decisions come from our earlier work [26, 24. In [26] we use discrete search to obtain a sequence of transitions that guides the generation of motions for a hybrid robotic system with 10-30 modes and mostly linear dynamics. In 24 we show that traditional motion-planning problems can be solved more efficiently by combining sampling-based motion planning with discrete search over an artificially imposed decomposition of the environment on which the robot moves (which in general can be regarded as a projection of its state space). The work presented in this paper combines and extends ideas developed in [26, 24 to obtain an effective testing method for hybrid systems with thousands of modes and nonlinear dynamics.

The proposed method, HyDICE, imposes a coarse-grained decomposition on the continuous state space associated with each mode. The decomposition and the discrete transitions of the hybrid system are used to construct a discrete search graph. Vertices of the search graph correspond to decomposition regions, while edges connect vertices corresponding to two adjacent decomposition regions or two decomposition regions that are connected by a discrete transition. The search graph is used to find leads, that is sequences of decomposition regions that constitute search directions and may be useful in finding a trajectory from a safe to an unsafe state. The search inside each of the continuous decomposition regions is done by a state-of-the-art sampling-based motion-planning technique. Information gathered during exploration, such as region coverage, exploration time and progress, and other quantities, are used to refine the discrete search and improve the quality of the lead computed for the next exploration step.

In contrast to previous work [15, 14, the multi-layered approach developed in this paper is well-suited for systems with many modes and transitions and offers considerable computational improvements over existing methods as demonstrated in this paper. Initial validation of HyDICE is provided by testing hybrid systems inspired by motion-planning problems that have thousands of modes and transitions and nonlinear dynamics associated with each mode. As indicated by the experiments, the tight integration of discrete search and exploration enables HyDICE to be up to two orders of magnitude faster than other related methods.

The rest of the paper is as follows. The hybrid-testing problem and the hybrid system used in the experiments are described in Section 2 Details of HyDICE are 
provided in Section 3. Experiments and results are presented in Section 4. We conclude in Section 5 with a discussion.

\section{Problem Description}

\subsection{Hybrid Automata and Hybrid-Testing Problem}

In this work, hybrid systems are modeled by hybrid automata 6]. A hybrid automaton is a tuple $H=(S, E, G, J, f, U, I, F)$, where $S=Q \times X ; Q$ is a discrete and finite set; each $X_{q} \in X, X_{q} \subseteq R^{\operatorname{dim}\left(\mathrm{X}_{\mathrm{q}}\right)}$, represents the continuous space associated with $q \in Q ; E \subseteq Q \times Q$ indicates discrete transitions; each $G_{\left(q_{i}, q_{j}\right)} \in G, G_{\left(q_{i}, q_{j}\right)} \subseteq X_{q_{i}}$, and each $J_{\left(q_{i}, q_{j}\right)} \in J, J_{\left(q_{i}, q_{j}\right)}: X_{q_{i}} \rightarrow X_{q_{j}}$, represent the guard set and reset function associated with $\left(q_{i}, q_{j}\right) \in E$, respectively. The continuous dynamics of the system in each $q \in Q$ is governed by a set of differential equations $f_{q}: X_{q} \times U_{q} \rightarrow \operatorname{Tg} X_{q}$, where $f_{q} \in f, U_{q} \subseteq R^{\operatorname{dim}\left(\mathrm{U}_{\mathrm{q}}\right)}$ denotes the set of possible input controls, and $\operatorname{Tg} X_{q}$ denotes the tangent space of $X_{q}$. Each $X_{q} \in X$ usually includes derivatives of different orders, e.g., velocity and acceleration of a car, and thus $f_{q}$ is typically nonlinear. The function $f_{q}$ has the form $f_{q}\left(x, u_{q}(x)\right)$, where the input $u_{q}(x) \in U_{q}$ associated with each $x \in X_{q}$ could represent continuous controls, nondeterminism, uncertainties, disturbances from the environment, or actions of other systems. In order to model these factors, the assignment $u_{q}(x) \in U_{q}$ could be non-deterministic or selected according to some probability distribution associated with $U_{q}$.

A hybrid system trajectory consists of one or more continuous trajectories interleaved with discrete transitions. Starting at some state $\left(q_{0}, x_{0}\right) \in I$, where $I \in S$ denotes the set of initial states, the system evolves according to $f_{q_{0}}$ until it reaches $G_{\left(q_{0}, q_{1}\right)}$, for some $q_{1} \in Q$. Then a discrete transition to $q_{1}$ occurs and the continuous state is reset by $J_{\left(q_{0}, q_{1}\right)}\left(x_{0}\right)$. The system evolution continues in such manner until the end of execution time.

A hybrid system is considered unsafe if a witness trajectory is produced that takes the hybrid system from some initial safe state $s_{\text {safe }} \in I$ to some $s_{\text {unsafe }} \in F$, where $F \subseteq S$ denotes a set of unsafe states.

\subsection{A Hybrid System Inspired by Motion Planning Problems}

The hybrid system used throughout this paper consists of an autonomous robotic car, whose underlying dynamics change discretely depending on terrain conditions. The choice of this specific system is to provide a concrete, scalable benchmark in which the competitiveness of our approach can be tested.

A given environment is divided into a number of terrains $\left\{R_{1}, \ldots, R_{N}\right\}$, where each $R_{i}$ corresponds to an operating mode $q_{i} \in Q$. The motion of the robotic car inside each terrain is specified by a set of ordinary differential equations. A discrete transition $\left(q_{i}, q_{j}\right)$ occurs when the robotic car enters a part of $R_{i}$ designated as the guard set $G_{\left(q_{i}, q_{j}\right)}$. The discrete transition indicates necessary changes in the way the robotic car should be controlled to adapt to changes in 
terrain conditions. After entering $R_{j}$, the continuous state of the robotic car is reset as specified by $J_{\left(q_{i}, q_{j}\right)}$ and the underlying dynamics of the car is specified according to the motion equations associated with $q_{j} \in Q$. The robotic car is said to have entered some unsafe state, for example, if it is in a particular terrain $R_{j}$ and the speed is above a certain predefined threshold. The robotic car could behave as a kinematic (first-order) car (KCar), smooth (second-order) car (SCar), smooth Reeds-Shepp car (RSCar), smooth unicycle (SUni), or smooth differential drive (SDDrive). Detailed descriptions of these models can be found in [17, 18,

Kinematic Car (KCar): A continuous state $x$ is of the form $x=[p, \theta]$, where $p \in$ $\mathbb{R}^{2}$ and $\theta \in(-\pi, \pi]$ denote the position and orientation of the robotic car. The motion equations are $\dot{p_{0}}=u_{0}(x) \cos (\theta) ; \dot{p_{1}}=u_{1}(x) \sin (\theta) ; \dot{\theta}=u_{0}(x) \tan \left(u_{1}(x)\right) /$ $L$, where $u_{0}(x) \in[-1,1]$ and $u_{1}(x) \in[-1,1]$ are the speed and steering wheel controls and $L$ is the distance between the front and rear axles.

Smooth Car (SCar): The kinematic car model can be extended to a second-order model by expressing the velocity $v$ and steering angle $\phi$ as differential equations of the acceleration $u_{0}(x)$ and the rotational velocity of the steering wheel $u_{1}(x)$ controls, as follows: $x=[p, \theta, v, \phi]$ and $\dot{p_{0}}=v \cos (\theta) ; \dot{p_{1}}=v \sin (\theta) ; \dot{\theta}=$ $v \tan (\phi) / L ; \dot{v}=u_{0}(x) ; \dot{\phi}=u_{1}(x)$.

Smooth Reeds-Shepp Car (RSCar): A smooth Reeds-Shepp car is similar to a smooth car, but the acceleration control $u_{0}(x)$ is only allowed to be from the set $\{-\max , 0, \max \}$, where $\max \in \mathbb{R}$ is some predefined parameter.

Smooth Unicycle (SUni): The continuous state $x$ is $x=[p, \theta, v, \omega]$, where $p, \theta$, $v$ are as in the smooth car model and $\omega$ indicates the rotational velocity. The motion equations are $\dot{p_{0}}=v \cos (\theta) ; \dot{p_{1}}=v \sin (\theta) ; \dot{\theta}=\omega ; \dot{v}=u_{0}(x) ; \dot{\omega}=u_{1}(x)$.

Smooth Differential Drive (SDDrive): The motion equations are $\dot{p_{0}}=0.5 r$ $\left(\omega_{\ell}+\omega_{r}\right) \cos (\theta) ; \dot{p_{1}}=0.5 r\left(\omega_{\ell}+\omega_{r}\right) \sin (\theta) ; \dot{\theta}=r\left(\omega_{r}-\omega_{\ell}\right) / L ; \dot{\omega}_{\ell}=u_{0}(x) ; \dot{\omega}_{r}=$ $u_{1}(x)$, where $x=\left[p, \theta, \omega_{\ell}, \omega_{r}\right]$ is the continuous state; $\omega_{\ell}$ and $\omega_{r}$ are the rotational velocities of the left and right wheels, respectively; $r$ is the wheel radius; and $L$ is the length of the axis connecting the centers of the two wheels.

The controls $u_{0}(x)$ and $u_{1}(x)$ could be thought of as playing the role of the automatic driver. The objective of hybrid-system testing is then to test the safety of the automatic driver, i.e., the driver is unsafe if a witness trajectory is produced that indicates that it is possible for the robotic car to enter an unsafe state. Due to length limitations of this paper, we only provide high-level descriptions of the automatic drivers 4 These driver models consist of simple ifthen-else statements depending on the state values and motion equations. In the first model, RandomDriver, $u_{0}(x)$ and $u_{1}(x)$ are selected pseudo-uniformly at random from some $\left[-\max _{0}, \max _{0}\right]$ and $\left[-\max _{1}, \max _{1}\right]$, respectively. In a second model, StudentDriver, the driver follows an approach similar to stop-and-go. When the speed is close to zero, StudentDriver selects $u_{0}(x)$ and $u_{1}(x)$ as in the RandomDriver model. Otherwise, StudentDriver selects controls that reduce the speed. The third model, HighwayDriver attempts to maintain the speed

\footnotetext{
4 See http://www.cs.rice.edu/CS/Robotics/CAV2007data/for more details.
} 
within acceptable low and upper bounds and avoid sharp turns. When the speed is too low, HighwayDriver selects controls that increase the speed. When the speed is too high, HighwayDriver selects controls that slow down the robotic car. When the speed is between the low and upper bounds, HighwayDriver selects controls that do not change the speed too much.

\section{Methods}

As discussed in the introduction, HyDICE constructs a discrete search graph based on the discrete transitions and a decomposition of the continuous state spaces. Observe that any witness trajectory from $s_{\text {safe }} \in I$ to $s_{\text {unsafe }} \in F$ passes through a sequence of decomposition regions. Although the converse does not hold, a sequence of connected decomposition regions, starting and ending in two regions containing states in $I$ and $F$, respectively, may contain a witness trajectory. Such sequences of connected regions, referred to as leads, provide search directions which are used by the sampling-based motion-planning method as guides for the exploration of the state space of a given hybrid system.

Algorithm 1. Pseudocode for HyDICE

Input: $H=(S, \operatorname{Inv}, E, G, J, f, U, I, F)$ : hybrid system; $t_{\max } \in \mathbb{R}$ : upper bound on overall computation time; $t_{e} \in \mathbb{R}$ : short time allocated to each exploration step

Output: A witness trajectory or FAILURE if no witness trajectory is found

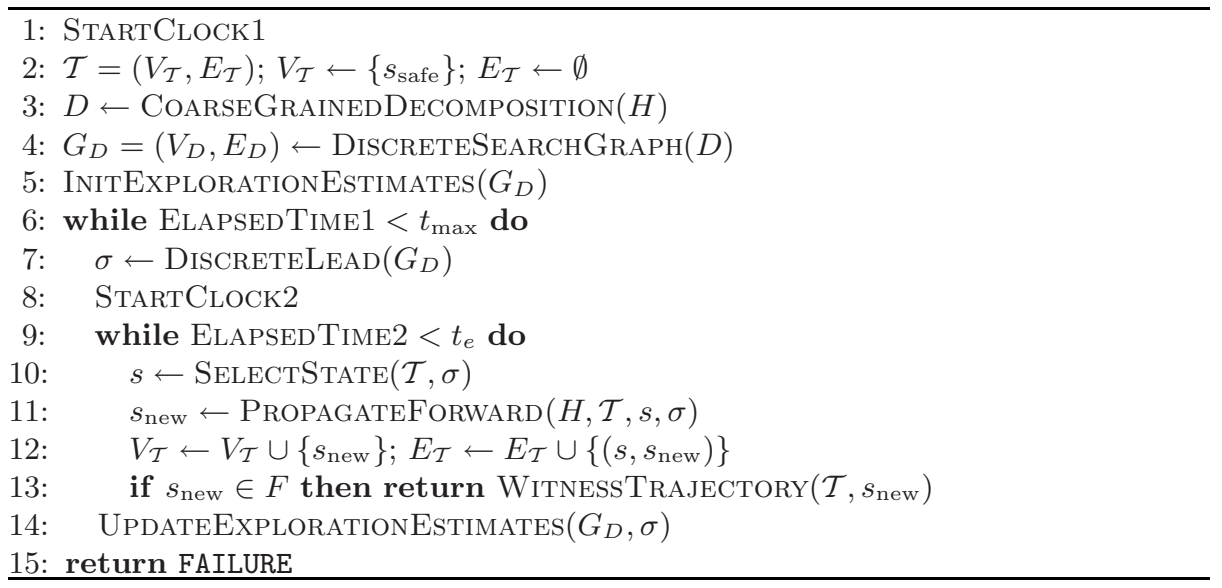

The search for a witness trajectory proceeds iteratively. Throughout the search, HyDICE maintains an exploration tree $\mathcal{T}=\left(V_{\mathcal{T}}, E_{\mathcal{T}}\right)$, which initially contains only $s_{\text {safe }}$, i.e., $V_{\mathcal{T}}=\left\{s_{\text {safe }}\right\}$ and $E_{\mathcal{T}}=\emptyset$. The vertices $V_{\mathcal{T}}$ are states of $S$, while an edge $\left(s^{\prime}, s^{\prime \prime}\right) \in E_{\mathcal{T}}$ indicates that a hybrid-system trajectory connects $s^{\prime} \in S$ to $s^{\prime \prime} \in S$. At each iteration, HyDICE uses the discrete search graph to compute a lead and then sampling-based motion planning to extend the branches of $\mathcal{T}$ in the direction specified by the lead. The branches of $\mathcal{T}$ are extended by adding new vertices and edges to $V_{\mathcal{T}}$ and $E_{\mathcal{T}}$, respectively. A witness trajectory 
is found when a state $s_{\text {unsafe }} \in F$ is added to $\mathcal{T}$. Otherwise, the search continues until an upper bound on computation time is exceeded. Pseudocode is provided in Algorithm 1. The discrete search and the sampling-based motion planning are described in Sections 3.1 and 3.2. respectively.

\subsection{Discrete Search}

Coarse-grained Decomposition. HyDICE constructs a coarse-grained decomposition $D=\left\{D_{q_{1}}, \ldots, D_{q_{N}}\right\}$, where $D_{q_{i}}=\left\{D_{q_{i}, 1}, \ldots, D_{q_{i}, n_{i}}\right\}$ denotes the decomposition of $X_{q_{i}}$ into $n_{i} \in \mathbb{N}$ different regions (line 3 of Algorithm 1 ). HyDICE does not impose any strict requirements on the decomposition. $D_{q_{i}}$ is usually computed as a set of nonoverlapping regions in some low-dimensional projection of $X_{q_{i}}$. For the hybrid system used in this work, HyDICE projects each $X_{q_{i}}$ onto $\mathbb{R}^{2}$ and constructs a cell-based decomposition. Other types of projections and decompositions are possible [17, 18].

Discrete Search Graph. $D$ is used to create a search graph $G_{D}=\left(V_{D}, E_{D}\right)$. A vertex $v_{q_{i}, j}$ is added to $V_{D}$ for each $D_{q_{i}, j}$. An edge $\left(v_{q_{i}, j}, v_{q_{i}, k}\right)$ is added to $E_{D}$ for each two adjacent regions $D_{q_{i}, j}$ and $D_{q_{i}, k}$. Furthermore, an edge $\left(v_{q_{i}, j}, v_{q_{\ell}, k}\right)$ is added to $E_{D}$ for each two regions $D_{q_{i}, j}$ and $D_{q_{\ell}, k}$ such that there is a discrete transition from some state $\left(q_{i}, x_{1}\right), x_{1} \in D_{q_{i}, j}$, to some state $\left(q_{\ell}, x_{2}\right)$, $x_{2} \in D_{q_{\ell}, k}$. There are also two special vertices $v_{\text {safe }}$ and $v_{\text {unsafe }}$ added to $V_{D}$. An edge $\left(v_{\text {safe }}, v_{q_{i}, j}\right)$ is added to $E_{D}$ for each $D_{q_{i}, j}$ such that $D_{q_{i}, j} \cap I \neq \emptyset$. Similarly, an edge $\left(v_{q_{i}, j}, v_{\text {unsafe }}\right)$ is added to $E_{D}$ for each $D_{q_{i}, j}$ such that $D_{q_{i}, j} \cap F \neq \emptyset$. This operation is found in line 4 of Algorithm 1.

Importance of Leads. A central issue is which lead to choose from the combinatorially large number of possibilities. This issue is addressed by associating a weight $w_{\left(v_{q_{i}, j}, v_{q_{\ell}, k}\right)}$ with each $\left(v_{q_{i}, j}, v_{q_{\ell}, k}\right) \in E_{D}$, which estimates the importance of including $\left(v_{q_{i}, j}, v_{q_{\ell}, k}\right) \in E_{D}$ as an edge in the lead. Preference is given to leads associated with higher edge weights. For the moment assume that there is no distinction between edges corresponding to discrete transitions and edges connecting adjacent regions in the decomposition. At the end we discuss the possibility of using different weighting schemes depending on the edge type.

Initially, the weights are set to a fixed value (line 5) and are updated (line 14) after each exploration step. The weight $w_{\left(v_{q_{i}, j}, v_{q_{\ell}, k}\right)}$ depends on the coverage of $D_{q_{i}, j}$ and $D_{q_{\ell}, k}$ by $\mathcal{T}$. The coverage $c\left(\mathcal{T}, D_{q_{i}, j}\right)$ is computed by imposing an implicit uniform grid on $D_{q_{i}, j}$ and measuring the fraction of cells that contain at least one state from $\mathcal{T}$. Let $c_{\text {prev }}\left(\mathcal{T}, D_{q_{i}, j}\right)$ denote $c\left(\mathcal{T}, D_{q_{i}, j}\right)$ at the beginning of the current exploration step (before line 9 ) and let $c_{\text {after }}\left(\mathcal{T}, D_{q_{i}, j}\right)$ denote $c\left(\mathcal{T}, D_{q_{i}, j}\right)$ at the end of the exploration step (after line 13). Thus $\Delta c\left(\mathcal{T}, D_{q_{i}, j}\right)=$ $c_{\text {after }}\left(\mathcal{T}, D_{q_{i}, j}\right)-c_{\text {prev }}\left(\mathcal{T}, D_{q_{i}, j}\right)$ indicates the change in the coverage of $D_{q_{i}, j}$ by $\mathcal{T}$ as a result of the current exploration step. $c\left(\mathcal{T}, D_{q_{\ell}, k}\right), c_{\text {prev }}\left(\mathcal{T}, D_{q_{\ell}, k}\right)$, $c_{\text {after }}\left(\mathcal{T}, D_{q_{\ell}, k}\right)$, and $\Delta c\left(\mathcal{T}, D_{q_{\ell}, k}\right)$ are defined similarly. Let $t$ denote the computation time devoted to the exploration of $D_{q_{i}, j}$ and $D_{q_{\ell}, k}$ during the current exploration step and let $t_{\text {acc }}\left(D_{q_{i}, j}, D_{q_{\ell}, k}\right)$ denote the accumulated exploration time devoted to $D_{q_{i}, j}$ and $D_{q_{\ell}, k}$. Then, the weight $w_{\left(v_{q_{i}, j}, v_{q_{\ell}, k}\right)}$ is defined as 
$w_{\left(v_{q_{i}, j}, v_{q_{\ell}, k}\right)}=(1-\epsilon)\left(\Delta c\left(\mathcal{T}, D_{q_{i}, j}\right)+\Delta c\left(\mathcal{T}, D_{q_{\ell}, k}\right)\right) /(2 t)+\epsilon / t_{\text {acc }}\left(D_{q_{i}, j}, D_{q_{\ell}, k}\right)$, where $0<\epsilon<1$ is a normalization constant. Large values of $w_{\left(v_{q_{i}, j}, v_{q_{\ell}, k}\right)}$ indicate promising leads, since such values are obtained when $\mathcal{T}$ in a short amount of time reaches previously uncovered parts of $D_{q_{i}, j}$ and $D_{q_{\ell}, k} \cdot t_{\text {acc }}(i, j)$ is used to increase the weight of those regions that have been explored less frequently.

As described so far the same procedure determines $w_{\left(v_{q_{i}, j}, v_{q_{\ell}, k}\right)}$, regardless of whether the edge from $D_{q_{i}, j}$ to $D_{q_{\ell}, k}$ corresponds to a discrete transition or adjacency in the decomposition. Intuitively, edges corresponding to discrete transitions may be more important, as they guide the sampling-based motion planner to extend branches of $\mathcal{T}$ from one continuous state space to another. For this reason, each $w_{\left(v_{q_{i}, j}, v_{q_{\ell}, k}\right)}$ corresponding to a discrete transition is multiplied by some constant $w>1$. Depending on the problem, it may also be beneficial to estimate the weights corresponding to discrete transitions differently.

Computation of Leads. Leads associated with higher edge weights are selected more frequently. At the same time, each lead has a non-zero probability of being selected. In this way, HyDICE aims to obtain a balance between greedy and methodical search. The computation of leads is essentially a graph-search problem and there is extensive literature on the subject 27. The approach undertaken in this work is to use combinations of different strategies, such as randomized depth-first search where the weights associated with each edge in $E_{D}$ are used to select the successor vertices in the search process, Dijkstra's algorithm, and other graph-search methods 27. For considerably larger problems, approaches from model checking, such as bounded model checking [28] or directed model checking 29, could also be used (see also discussion in Section 5).

DiscreteLeAD $\left(G_{D}\right)$ (line 7 ) returns more frequently the most probable lead and the lead associated with the highest sum of edge weights and less frequently leads computed by randomized depth-first search. The most probable lead is computed using Dijkstra's algorithm and setting the weight function used in the graph search to $-\log \left(w_{\left(v_{q_{i}, j}, v_{q_{\ell}, k}\right)} / w_{\text {total }}\right)$ for $\left(v_{q_{i}, j}, v_{q_{\ell}, k}\right) \in E_{D}$, where $w_{\text {total }}=$ $\sum_{\left(v^{\prime}, v^{\prime \prime}\right) \in E_{D}} w_{\left(v^{\prime}, v^{\prime \prime}\right)}$. The lead with the highest sum of edge weights is computed using Dijkstra's algorithm and setting the weight function to $w_{\max }-w_{\left(v_{q_{i}, j}, v_{q_{\ell}, k}\right)}$ for $\left(v_{q_{i}, j}, v_{q \ell, k}\right) \in E_{D}$, where $w_{\max }$ denotes the maximum weight.

\subsection{Sampling-Based Motion Planning}

The objective of the exploration step (lines 9-13) is to use the lead $\sigma$ to extend $\mathcal{T}$ toward $F$. The exploration step proceeds iteratively by selecting a state $s$ from $\mathcal{T}$ and propagating forward from $s$ to a new state $s_{\text {new }}$.

Conceptually, forward propagation provides the necessary mechanism for sampling-based motion planning to extend the branches of $\mathcal{T}$ and explore the state space. The forward propagation from $s$ entails simulating the evolution of $H$ starting at $s$ and for a duration of $t$ units of time, where $t$ is selected pseudo-uniformly at random from $\left[\min _{t}, \max _{t}\right] \subset(0, \infty)$. The simulation can be computed by numerically integrating the motion equations for a short period of time and following the appropriate discrete transitions when guard conditions 
are met. The simulation terminates if at any point an unsafe state is reached (see 14 for more details). The new state $s_{\text {new }}$ obtained at the end of the simulation and the edge $\left(s, s_{\text {new }}\right)$ are added to the vertices and edges of $\mathcal{T}$, respectively.

$\operatorname{SelectState}(\mathcal{T}, \sigma)$ (line 10$)$ selects more frequently states $s$ from $\mathcal{T}$, which, when propagated forward, bring $\mathcal{T}$ closer to $F$. Let $D_{q_{i_{1}}, j_{1}}, \ldots, D_{q_{i_{n}}, j_{n}}$ be the coarse-grained decomposition regions associated with the sequence of vertices $v_{\text {safe }}, v_{q_{i_{1}}, j_{1}}, \ldots, v_{q_{i_{n}}, j_{n}}, v_{\text {unsafe }}$ in $\sigma$. Since $\sigma$ is a sequence of edges from $v_{\text {safe }}$ to $v_{\text {unsafe, }}$, the order $1 \leq k \leq n$ in which $v_{q_{i_{k}}, j_{k}}$ appears in $\sigma$ provides an indication of how close $D_{q_{i_{k}}, j_{k}}$ is to $F$. Since the objective of the exploration step is to extend branches of $\mathcal{T}$ closer to $F$, HyDICE gives preference to regions $D_{q_{i_{k}}, j_{k}}$ that are closer to $F$, i.e., $k$ is close to $n$. To balance this greedy approach, HyDICE also takes into account the overall exploration time $t_{\text {acc }}\left(D_{q_{i_{k}}, j_{k}}\right)$ spent in each $D_{q_{i_{k}}, j_{k}}$ and the coverage $c\left(\mathcal{T}, D_{q_{i_{k}}, j_{k}}\right)$. If $D_{q_{i_{k}}, j_{k}}$ contains states from $\mathcal{T}$, let $w_{k}=$ $\alpha k / n+\beta / c\left(\mathcal{T}, D_{q_{i_{k}}, j_{k}}\right)+\gamma / t_{\mathrm{acc}}\left(D_{q_{i_{k}}, j_{k}}\right)$, where $\alpha, \beta$, and $\gamma$ are normalization constants. Otherwise, let $w_{k}=0 . \operatorname{SeLECTState}(\mathcal{T}, \sigma)$ selects a region $D_{q_{i_{k}}, j_{k}}$ with probability $w_{k} / \sum_{h=1}^{m} w_{h}$. Each state $s$ from $\mathcal{T}$ that is contained in $D_{q_{i_{k}}, j_{k}}$ is selected with probability $1 / \operatorname{nsel}(s)$, where $\operatorname{nsel}(s)$ is the number of times $s$ has been previously selected. Preference is thus given to states that have been selected less frequently, since such states, when propagated forward, can cause $\mathcal{T}$ to extend in previously unexplored directions.

PropagateForward $(H, \mathcal{T}, s, \sigma)$ attempts to extend $s$ toward $D_{q_{i_{k+1}}, j_{k+1}}$ and thus bring $\mathcal{T}$ closer to $F$. Since the evolution of $H$ can be nondeterministic, PropagateForward $(H, \mathcal{T}, s, \sigma)$ tries several times to propagate forward from $s$. Let $s_{\text {new }_{i}}$ be the state obtained after simulating the evolution of $H$ from $s$ for a duration of $t_{i}$ units of time, where $t_{i}$ is selected pseudo-uniformly at random from $\left[\min _{t}, \max _{t}\right]$. PropagateForward $(H, \mathcal{T}, s, \sigma)$ computes $s_{\text {new }}$ as the state $s_{\text {new }_{i}}$ that is the closest to $D_{q_{i_{k+1}}, j_{k+1}}$. A witness trajectory is found if $s_{\text {new }} \in F$. The witness trajectory is computed by reconstructing the evolution of the hybrid system from $s_{\text {safe }}$ to $s_{\text {new }}$ following the appropriate edges of $\mathcal{T}$ (line 13).

\section{Experiments and Results}

Experiments are performed using the hybrid robotic system described in Section 2.2. The hybrid robotic system is made increasingly complex by increasing the number of modes. This paper presents experiments with up to 10000 modes.

An important part of experiments is the comparison with previous related work. The closest work we can compare to is the application of RRT to hybrid systems [14, 15. We also provide experiments that indicate the impact of the discrete search on the computational efficiency of HyDICE.

A problem instance is obtained by fixing the number $N$ of operating modes of the hybrid robotic car. The continuous dynamics associated with each mode $q_{i}$ (or terrain $R_{i}$ ) is selected pseudo-uniformly at random from KCar, SCar, RSCar, SUni, and SDDrive. The set of discrete transitions $E$ is created as follows. Initially, discrete transitions are added between each pair $R_{i}, R_{j}$ of neighboring 
terrains. A disjoint-set strategy, similar to maze creation, is then used to remove certain discrete transitions. Furthermore, each remaining discrete transition is kept with probability $p$. We experimented with different values of $p$ and found that it has minimal impact on the comparisons between HyDICE and RRT. Experiments reported in this paper use $p=0.1$. For each problem instance, we create 30 safety properties. Each safety property is created by selecting pseudouniformly at random one terrain as safe and another one as unsafe. As discussed in Section 2.2, the hybrid robotic car is said to have entered some unsafe state, if it is in an unsafe terrain and its speed is above a certain predefined threshold. In all the experiments, the systems were unsafe. For safe systems, since the hybrid-system testing problem is generally undecidable, all the tools used in the experiments would timeout.

Results. For each problem instance, experiments are run using each of the driver models. Results are summarized in Table 1. We report the average computational time in seconds required by RRT, HyDICE*, and HyDICE to test 30 safety properties. HyDICE* refers to the version of HyDICE that does not use the discrete-search component, i.e., HyDICE* is the sampling-based motion planner of HyDICE with some minor modifications 5 Comparisons include HyDICE* to investigate the importance of the discrete search on HyDICE. An entry marked with $X$ indicates that the testing method timed out. The upper bound on time was set to $3000 \mathrm{~s}$ for each safety property testing. The time allocated to each exploration step by HyDICE $\left(t_{e}\right.$ in Algorithm 1) was set to 1s. The Rice PBC Cluster and Rice Cray XD1 Cluster ADA were used for code development. Experiments were run on ADA, where each processor runs at $2.2 \mathrm{GHz}$ and has up to $8 \mathrm{~GB}$ of RAM.

Table 1. Summary of experimental comparisons. Time is in seconds

\begin{tabular}{r|ccc|ccc|ccc}
\multicolumn{4}{c}{ RandomDriver } & \multicolumn{3}{c}{ StudentDriver } & \multicolumn{3}{c}{ HighwayDriver } \\
$|Q|$ & RRT & HyDICE* HyDICE & RRT & HyDICE* HyDICE & RRT & HyDICE* HyDICE \\
\hline 100 & 22.30 & 4.34 & 2.68 & 74.01 & 6.74 & 2.20 & 21.29 & 4.92 & 2.82 \\
225 & 117.79 & 14.02 & 6.24 & 336.85 & 32.44 & 5.24 & 230.88 & 21.64 & 6.30 \\
525 & 295.88 & 75.60 & 6.87 & 792.45 & 65.40 & 15.52 & 668.67 & 106.56 & 16.31 \\
900 & 504.93 & 175.96 & 13.74 & $\mathrm{X}$ & 120.48 & 17.06 & 2596.50 & 182.54 & 36.96 \\
1600 & 2159.24 & 289.94 & 32.52 & $\mathrm{X}$ & 464.56 & 34.14 & $\mathrm{X}$ & 374.44 & 37.26 \\
2500 & $\mathrm{X}$ & 910.86 & 60.18 & $\mathrm{X}$ & 699.66 & 62.30 & $\mathrm{X}$ & 929.36 & 71.44 \\
10000 & $\mathrm{X}$ & $\mathrm{X}$ & 439.88 & $\mathrm{X}$ & $\mathrm{X}$ & 457.60 & $\mathrm{X}$ & $\mathrm{X}$ & 445.52
\end{tabular}

Table 1 shows that HyDICE is consistently more efficient than RRT. When the RandomDriver model is used, HyDICE is 8.32, 18.87, 43.06, and 66.39 times faster than RRT, as the number of modes is increased to $100,225,525$, and 1600 , respectively. Furthermore, RRT times out when $|Q|=2500$, while HyDICE requires only 60.18s. Similarly, when the StudentDriver model is used, the computational

\footnotetext{
${ }^{5}$ HyDICE* can be obtained from the implementation of HyDICE by computing the lead $\sigma$ as $v_{\text {safe }}, \gamma, v_{\text {unsafe, }}$, where $\gamma$ is a random permutation of $V_{D}-\left\{v_{\text {safe }}, v_{\text {unsafe }}\right\}$, where, as described in Section 3. $G_{D}=\left(V_{D}, E_{D}\right)$ is the search graph.
} 
speedups obtained by HyDICE vary from 33.64 to 51.05 on instances where RRT does not time out. Under the StudentDriver model, RRT times out on instances with $|Q|=900$, while HyDICE requires only 17.06s. The StudentDriver model is particularly computationally challenging for RRT since the stop-and-go approach it uses makes it difficult for RRT to extend the exploration tree. On the other hand, since HyDICE relies on a discrete search component it successfully extends the exploration tree and quickly reaches unsafe states. Similar observations are made for the HighwayDriver model as well.

Table 1 indicates that HyDICE is up to two orders of magnitude computationally faster than RRT. Table 1 also shows that HyDICE scales up reasonably well with respect to $|Q|$. In fact, RRT timed out in all cases when $|Q| \geq 2500$, while HyDICE is shown to handle problems even with $|Q|=10000$ quite efficiently.

The second set of experiments provides insight on the observed computational efficiency of HyDICE. In particular, we investigate the importance of the discrete search on HyDICE. Table 1 shows that although HyDICE* is still faster than RRT, it is considerably slower than HyDICE. (For a discussion on issues related to the computational efficiency of RRT and sampling-based motion planners similar to HyDICE* see [17, 18, 21, 24.) For example, when $|Q|=2500$, HyDICE* is 11-15 times slower than HyDICE. Furthermore, HyDICE* times out on instances with $|Q|=10000$, while HyDICE handles such instances efficiently. These results highlight the importance of the discrete search and agree with observations made in 24. The interplay between lead computations and sampling-based exploration has the desired effect of quickly improving the quality of future leads and explorations and bringing the search closer to obtaining a solution. By guiding the exploration, the discrete search significantly improves the computational efficiency of HyDICE.

\section{Discussion}

We have presented HyDICE, a multi-layered approach for hybrid-system testing that blends sampling-based motion planning with discrete searching. The discrete search, responsible for managing the potentially huge complexity of discrete transitions, also uses coarse-grained decompositions of the continuous state spaces or related projections to guide the motion planner during the search for witness trajectories. The motion planner feeds back to the discrete search information gathered during the exploration, which is then used to further refine the discrete search and guide the motion planner toward increasingly promising search directions. This tight integration of discrete search and motion planning in the framework of HyDICE offers considerable computational advantages over related work. Experiments presented in this paper, using a hybrid robotic car, different driving models, and nonlinear dynamics associated with each of the several thousand modes, provide initial validation of HyDICE and demonstrate its promise as a hybrid-system testing method. Comparisons to related work show computational speedups of up to two orders of magnitude. 
Although HyDICE was shown to handle a system with thousands of modes and nonlinear dynamics, the scalability issue is relevant and remains open to further research. As the number of modes becomes significantly large, the simple graphsearch strategies used in this work becomes a computational bottleneck and need to be replaced with state-of-the-art techniques developed in the verification community which can handle discrete systems with billions of modes 30 .

Additionally, the search graph is based on a decomposition of the continuous state spaces or related projections and the ability to determine whether or not two decomposition regions are connected by a discrete transition. Depending on the hybrid system, guard sets, and reset functions it may be challenging to determine if such discrete transition exists. In such cases, a viable approach would be to resort to approximations of guard sets and reset functions, which also requires investigating the overall impact of approximations on HyDICE.

One important theoretical issue that is subject of ongoing research relates to guarantees HyDICE can offer for general hybrid-system testing. Although completeness cannot be guaranteed, since the problem is generally undecidable, our belief is that HyDICE offers a weaker form of completeness, referred to as probabilistic completeness. Guaranteeing probabilistic completeness means that, for unsafe systems, the probability of finding a witness trajectory goes to one as the running time approaches infinity [17. Probabilistic completeness allows us to increase the confidence in the safety of the system as the running time increases. The work in 31 has already proven probabilistic completeness in a continuous setting for certain classes of motion-planning methods, such as the one used by HyDICE. The theoretical framework developed in [31] is also promising for showing probabilistic completeness in a hybrid-system setting and we are currently investigating such an approach.

We also intend in future work to experiment with HyDICE in other settings and apply it to increasingly realistic and complex hybrid systems.

\section{Acknowledgment}

This work has been supported in part by NSF CNS 0615328 (EP, LEK, MYV), NSF 0308237 (EP, LEK), a Sloan Fellowship (LEK), and NSF CCF 0613889 (MYV). Experiments were obtained on equipment supported by NSF CNS 0454333 and NSF CNS 0421109 in partnership with Rice University, AMD, and Cray.

\section{References}

1. Glover, W., Lygeros, J.: A stochastic hybrid model for air traffic control simulation. In: Alur, R., Pappas, G.J. (eds.) HSCC 2004. LNCS, vol. 2993, pp. 372-386. Springer, Heidelberg (2004)

2. Pepyne, D., Cassandras, C.: Optimal control of hybrid systems in manufacturing. Proceedings of IEEE 88(7), 1108-1123 (2000)

3. Johansson, R., Rantzer, A. (eds.): Nonlinear and Hybrid Systems in Automotive Control. Springer, London, UK (2003) 
4. Dounias, G., Linkens, D.A.: Adaptive systems and hybrid computational intelligence in medicine. Artificial Intelligence in Medicine 32(3), 151-155 (2004)

5. Piazza, C., Antoniotti, M., Mysore, V., Policriti, A., Winkler, F., Mishra, B.: Algorithmic algebraic model checking I: Challenges from systems biology. In: Etessami, K., Rajamani, S.K. (eds.) CAV 2005. LNCS, vol. 3576, pp. 5-19. Springer, Heidelberg (2005)

6. Alur, R., Courcoubetis, C., Henzinger, T., Ho, P.H.: Hybrid automata: an algorithmic approach to the specification and verification of hybrid systems. In: Grossman, R.L., Ravn, A.P., Rischel, H., Nerode, A. (eds.) Hybrid Systems. LNCS, vol. 736, pp. 209-229. Springer, Heidelberg (1993)

7. Henzinger, T., Kopke, P., Puri, A., Varaiya, P.: What's decidable about hybrid automata? In: STOC, pp. 373-382. ACM Press, New York (1995)

8. Henzinger, T.: The theory of hybrid automata. In: Proc. 11th IEEE Symp. on Logic in Computer Science, DIMACS, pp. 278-292. IEEE Computer Society Press, Los Alamitos (1996)

9. Lafferriere, G., Pappas, G., Yovine, S.: A new class of decidable hybrid systems. In: Vaandrager, F.W., van Schuppen, J.H. (eds.) HSCC 1999. LNCS, vol. 1569, pp. 137-151. Springer, Heidelberg (1999)

10. Puri, A.: Theory of Hybrid Systems and Discrete Event Systems. PhD thesis, University of California, Berkeley (1995)

11. Tomlin, C.J., Mitchell, I., Bayen, A., Oishi, M.: Computational techniques for the verification and control of hybrid systems. In: Proc. of IEEE, vol. 91(7), pp. 9861001 (2003)

12. Chutinan, C., Krogh, B.H.: Computational techniques for hybrid system verification. IEEE Transactions on Automatic Control 48(1), 64-75 (2003)

13. Silva, B., Stursberg, O., Krogh, B., Engell, S.: An assessment of the current status of algorithmic approaches to the verification of hybrid systems. In: IEEE Conf. on Decision and Control. vol. 3, pp. 2867-2874 (2001)

14. Esposito, J.M., Kim, J., Kumar, V.: Adaptive RRTs for validating hybrid robotic control systems. In: WAFR, Zeist, Netherlands, PP. 107-132 (2004)

15. Kim, J., Esposito, J.M., Kumar, V.: An RRT-based algorithm for testing and validating multi-robot controllers. In: RSS, Boston, MA, 249-256 (2005)

16. Copty, F., Fix, L., Fraer, R., Giunchiglia, E., Kamhi, G., Tacchella, A., Vardi, M.: Benefits of bounded model checking at an industrial setting. In: Berry, G., Comon, H., Finkel, A. (eds.) CAV 2001. LNCS, vol. 2102, pp. 436-453. Springer, Heidelberg (2001)

17. Choset, H., Lynch, K.M., Hutchinson, S., Kantor, G., Burgard, W., Kavraki, L.E., Thrun, S.: Principles of Robot Motion: Theory, Algorithms, and Implementations. MIT Press, Cambridge, MA (2005)

18. LaValle, S.M.: Planning Algorithms. Cambridge University Press, Cambridge (2006)

19. LaValle, S.M., Kuffner, J.J.: Rapidly-exploring random trees: Progress and prospects. In: Donald, B.R., Lynch, K., Rus, D. (eds.) WAFR, pp. 293-308 (2000)

20. Hsu, D., Kindel, R., Latombe, J.C., Rock, S.: Randomized kinodynamic motion planning with moving obstacles. IJRR 21(3), 233-255 (2002)

21. Plaku, E., Bekris, K.E., Chen, B.Y., Ladd, A.M., Kavraki, L.E.: Sampling-based roadmap of trees for parallel motion planning. IEEE Trans. on Robotics 21(4), 597-608 (2005)

22. Ladd, A.M., Kavraki, L.E.: Motion planning in the presence of drift, underactuation and discrete system changes. In: RSS, Boston, MA, pp. 233-241 (2005) 
23. Bekris, K.E., Kavraki, L.E.: Greedy but safe replanning under kinodynamic constraints. In: IEEE ICRA, Rome, Italy (2007)

24. Plaku, E., Vardi, M.Y., Kavraki, L.E.: Discrete search leading continuous exploration for kinodynamic motion planning. In: RSS, Atlanta, GA (2007)

25. Kavraki, L.E., Švestka, P., Latombe, J.C., Overmars, M.H.: Probabilistic roadmaps for path planning in high-dimensional configuration spaces. IEEE Transactions on Robotics and Automation 12(4), 566-580 (1996)

26. Plaku, E., Kavraki, L.E., Vardi, M.Y.: A motion planner for a hybrid robotic system with kinodynamic constraints. In: IEEE ICRA, Rome, Italy (2007)

27. Zhang, W.: State-space Search: Algorithms, Complexity, Extensions, and Applications. Springer, New York (2006)

28. Biere, A., Cimatti, A., Clarke, E., Fujita, M., Zhu, Y.: Symbolic model checking using SAT procedures instead of BDDs. In: Proc. 36th Design Automation Conference, pp. 317-320. IEEE Computer Society Press, Los Alamitos (1999)

29. Edelkamp, S., Jabbar, S.: Large-scale directed model checking ltl. In: Valmari, A. (ed.) Model Checking Software. LNCS, vol. 3925, pp. 1-18. Springer, Heidelberg (2006)

30. Burch, J., Clarke, E., McMillan, K., Dill, D., Hwang, L.: Symbolic model checking: $10^{20}$ states and beyond. Information and Computation 98(2), 142-170 (1992)

31. Ladd, A.M.: Motion Planning for Physical Simulation. PhD thesis, Rice University, Houston, TX (2006) 